\title{
Analysis of EEG Signal Processing Techniques based on Spectrograms
}

\author{
Ricardo Ramos-Aguilar, J. Arturo Olvera-López, Ivan Olmos-Pineda \\ Benemérita Universidad Autónoma de Puebla, \\ Faculty of Computer Science, Puebla, Mexico \\ ricramosal@gmail.com, \{aolvera, iolmos\}@cs.buap.mx
}

\begin{abstract}
Current approaches for the processing and analysis of EEG signals consist mainly of three phases: preprocessing, feature extraction, and classification. The analysis of EEG signals is through different domains: time, frequency, or time-frequency; the former is the most common, while the latter shows competitive results, implementing different techniques with several advantages in analysis. This paper aims to present a general description of works and methodologies of EEG signal analysis in time-frequency, using Short Time Fourier Transform (STFT) as a representation or a spectrogram to analyze EEG signals.
\end{abstract}

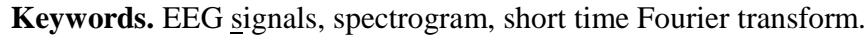

\section{Introduction}

The human brain is one of the most complex organs in the human body. It is considered the center of the human nervous system and controls different organs and functions, such as the pumping of the heart, the secretion of glands, breathing, and internal temperature. Cells called neurons are the basic units of the brain, which send electrical signals to control the human body and can be measured using Electroencephalography (EEG).

Electroencephalography measures the electrical activity of the brain by recording via electrodes placed either on the scalp or on the cortex. These time-varying records produce potential differences because of the electrical cerebral activity. The signal generated by this electrical activity is a complex random signal and is non-stationary [1]. Signals are normally composed of four brainwave groups: Delta, Theta, Alpha, and Beta [2]. Each band has different frequencies and amplitudes related to activities in the human body.

Currently, the analysis of EEG signals is very important due to the information obtained from the signal, which can help physicians to recognize brain issues such as epilepsy, Alzheimer's, seizure disorder, attention deficit disorder, anxiety disorder, fetal alcohol syndrome, and autism, among others [3,4,5,6,7]. The collected data also allows the brain to interact with machines or devices without physical contact, a process commonly called Brain Computer-Interfaces (BCI), which is the basis for different 
applications, e.g., assistive technologies for those with severe motor disabilities [8,9]. Finally, EEG signals are proposed as an alternative to biometric applications, as they are unique and cannot be forged [7].

There are different approaches to analyzing EEG with two of the most common being time and frequency domain. However, this analysis is not unique since it depends of the application; besides, considering that non-stationary signals are considered and different artifacts are added, classifying mental stages is not a trivial problem with opportunity to do research in this area. Another problem with a big challenge is dimensionality, since the information obtained for analysis, does not usually use only a few channels of acquisition[10]. Nevertheless, not all the information obtained is useful; it could present redundant data and increase computational time [3]. Feature extraction and selection can reduce the data dimension, in addition to showing relevant patterns of EEG signals associated with the brain activity, which may reflect good performance in classification [11,12], showing other opportunity area to research.

This paper analyzes various techniques applied for processing EEG signals, showing their characteristics mainly spectrograms-based methods. The outline of this paper is as follows: The phases of EEG analysis are discussed in the following section. An overview of reviewed works is presented in the Discussion section. Finally, conclusions are stated in the last section.

\section{EEG Signal Analysis Based on Spectrograms}

EEG signal analysis is commonly based on three modules or phases: Artifact removal or preprocessing, Feature Extraction, and Classification, as shown in Figure 1. The first phase is preprocessing, which cleans the signal of artifacts stored during acquisition; the next phase, feature extraction, retrieves the relevant features from a previously obtained spectrogram when SSTF is applied to the clean signal; finally, in the last phase, features are provided to classifiers to construct a model for the analysis of future cases.

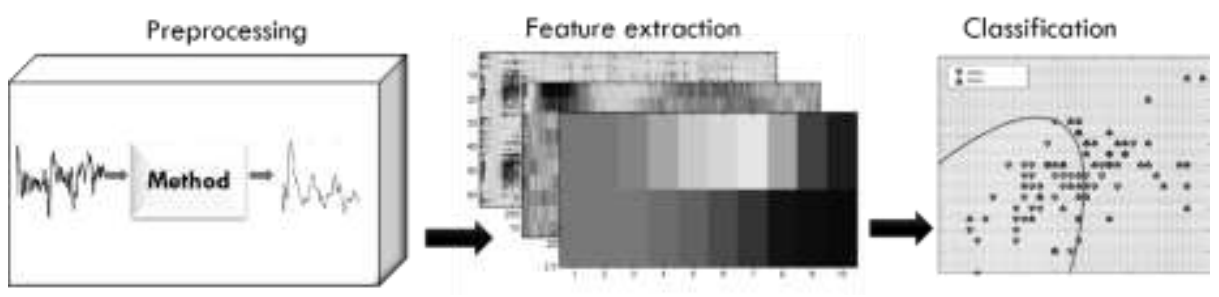

Fig. 1. Phases of EEG Signal Analysis.

\subsection{Preprocessing}

The preprocessing phase is the first step, which has two objectives: remove artifacts and filter data such as blinking, heartbeats, and other effects [4,13]. These can be implemented in the acquisition (phase) through hardware or by software-based techniques. Some well-known techniques for preprocessing EEG signals are Common Spa- 
tial Patterns (CSP), Principle Component Analysis (PCA), Common Average Referencing (CAR), Surface Laplacian(SL), Independent Component Analysis(ICA), Adaptive Filtering, Digital Filter, and others [4,14].

The approaches based on CSP construct spatial filters that both maximize the variance of one task and minimize the variance of another [4]. CSP is a discriminative userspecific filtering technique for enhancing the signal, which detects patterns within the EEG signals by incorporating the spatial information of the EEG. It uses covariance matrices as the basis and seek a discriminative subspace, such that the variance for one class is maximized and the variance of the other is minimized simultaneously [14]. PCA converts a number of correlated variables into a set of values of uncorrelated variables called principal components.

The primary axis or the first principal component is calculated such that it accounts for the largest amount of variability in the data. Subsequent components are calculated and account for the direction of the remaining variability, but in decreasing order of the amount of variability in the data, representing the direction of the next largest variation. As the transformed data have most of the variation in the first components, the remaining components can be ignored to decrease the dimensionality [14]; CAR removes the noise by subtracting the common activity from the position of interest to improve the Signal-to-Noise Ratio (SNR), which measures the EEG signal strength relative to background noise. SL is a method with the same objective as CAR: to improve SNR and allow it to view the EEG signal with a high spatial resolution $[4,14]$. The CAR method re-references the signal to a common average across all the neurosensors by subtracting the mean value of the signal in all electrodes from each sample. This mean represents an estimate of the activity at the reference site, and subtracting this average produces a de-referenced solution.

Contrary to CAR, the Surface Laplacian method is derived at a specific electrode position by subtracting a combination of the signals from a set of electrodes surrounding the central electrode signal [5]; ICA is a statistical and computational method for revealing the hidden sources/components that underlie sets of random variables, such as EEG signals. ICA assumes that the unknown underlying sources are independent of each other and have been linearly combined to form a mixed signal. This returns the independent components when this independence assumption is correct [5]. Adaptive filtering can modify signal properties according to the specific characteristics of the EEG, an efficient method for solving the problem of signals and interferences with overlapping spectra [14]. Digital filters are commonly used in artifact processing, where filters are implemented as low-pass, high-pass, band-pass, and band stop; these need to select the appropriate frequency to filter noise and artifacts [4]. Table 1 shows different characteristics of the methods described in [4,14]; most of these methods focus on temporal (CSP, CAR, PCA, SL, ICA) and spatial (Fourier Analysis, Autoregressive) filters. One important difference between filters in the response during online analysis is the time, as spatial filtering is better than temporal filtering with regard to time [4]. These different methods depend on the application, data dimension, and technology used in acquisition. 
Table 1. Characteristics of preprocessing methods.

\begin{tabular}{|c|c|}
\hline Method & Characteristics \\
\hline CAR & $\begin{array}{l}\text { Improves SNR and outperforms all reference methods; incomplete coverage causes } \\
\text { mistakes. }\end{array}$ \\
\hline ICA & $\begin{array}{l}\text { Computationally efficient and high performing for large data sizes; cannot be applied } \\
\text { to some cases and requires more calculations for decomposition. }\end{array}$ \\
\hline SL & $\begin{array}{l}\text { Robust against artifacts generated at regions uncovered by electrodes, sensitive to } \\
\text { artifacts and spline patterns. }\end{array}$ \\
\hline PCA & Reduces dimension and works better than ICA. \\
\hline CSP & Processes correctly motor imagery data and needs multiple electrodes (>64) \\
\hline Adaptive Filter & $\begin{array}{l}\text { Works well for signals with overlapping spectra and needs two signals and one refer- } \\
\text { ence signal. }\end{array}$ \\
\hline Digital Filter & Easily removes noise, requires multiple frequencies. \\
\hline
\end{tabular}

\subsection{Feature Extraction and Classification}

The second phase in EEG signal analysis is feature extraction, where features of the signal are obtained using different signal processing techniques, such as Fast Fourier Transform (FFT), Principal Component Analysis (PCA), Wavelet Transformations (WT), Auto Regressive (AR), and others [4]. Analysis in time or frequency domain offers only time/frequency and amplitude information. The aforementioned techniques are commonly used in both domains. The Time-Frequency domain allows extracting information in the two domains simultaneously; EEG analysis is based on the timefrequency image processing technique or spectrogram, a technique commonly used in Short Time Fourier Transform, which maps the signal into a two-dimensional function of frequency and time [2]. This section shows a review of the literature on extracting features of EEG signals using STFT.

EEG signals in time-frequency domain are retrieved using the spectrogram, by applying a Short Time Fourier Transform to the signal. STFT is applied to partition the EEG signal into several segments of short-time signals by shifting the time window with some overlapping [15], a process called windowing. Depending on the time windowing function $w[\mathrm{n}]$, a spectrogram is classified as a narrowband or wideband. If the time window is short, then its Fourier transform will be a wideband and a longer time returns a narrowband spectrogram [16]. The STFT general equation of a signal $S$ is given by equation (1):

$$
\mathrm{S}(\mathrm{m}, \mathrm{k})=\sum_{\mathrm{n}=0}^{\mathrm{N}-1} \mathrm{~s}\left(\mathrm{n}+\mathrm{mN}^{\prime}\right) \mathrm{w}(\mathrm{n}) \mathrm{e}^{-\mathrm{j} \frac{2 \pi}{\mathrm{N}} \mathrm{nk}},
$$

where

$k=[0: K]$ is the $\mathrm{k}^{\text {th }}$ Fourier coefficient.

$K=N / 2$ is the frequency index corresponding to the Nyquist frequency.

$S(m, k)$ indicates the m-index time-frequency(frame) spectrogram.

$N=$ window segment length.

$N^{\prime}=$ the shifting step of the time window. 
$w(n)=$ windowing method of an $\mathrm{N}$ - point sequence.

$N$ ' should be smaller than $\mathrm{N}$ in order to produce an overlap between the time windows. $S$ depends on the window function; in practice, different window shapes are used, such as: Symmetric, Unimodal and Gaussian.

The spectrogram contains a compromise between time resolution and frequency resolution: a large window provides less localization in time and more discrimination in frequency. The window obtains a time-slice of the signal, during which the spectral characteristics are nearly constant [16]; the obtained segments shift the time window with some overlapping. The spectrogram is defined as the magnitude of $S(m, k)$, represented as $A(m, k)$, as show in equation (2):

$$
\mathrm{A}(\mathrm{m}, \mathrm{k})=\frac{1}{\mathrm{~N}}|\mathrm{~S}(\mathrm{~m}, \mathrm{k})|^{2} .
$$

The spectrogram resolution can be enhanced modifying the length window; a long window provides a better frequency resolution, but poor time resolution. A short window, however, provides better time resolution but poor frequency resolution. A good visualization in the spectrogram depends the selection of an appropriate window length and overlapping. Fig. 2 shows a spectrogram of a signal, which is a time-varying spectral representation of a signal. A spectrogram layout is usually as follows: the $\mathrm{x}$-axis represents time, the y-axis represents frequency, and the third dimension is amplitude (spectral content) of a frequency-time pair, which is color coded. This three dimensional data can also create a 3D plot, where the intensity is represented as height on the $\mathrm{z}$-axis but a $2 \mathrm{D}$ chart provides a better understanding.

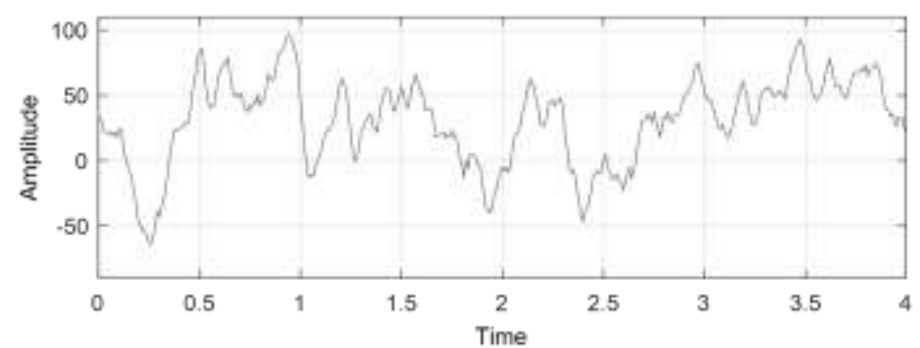

(a)

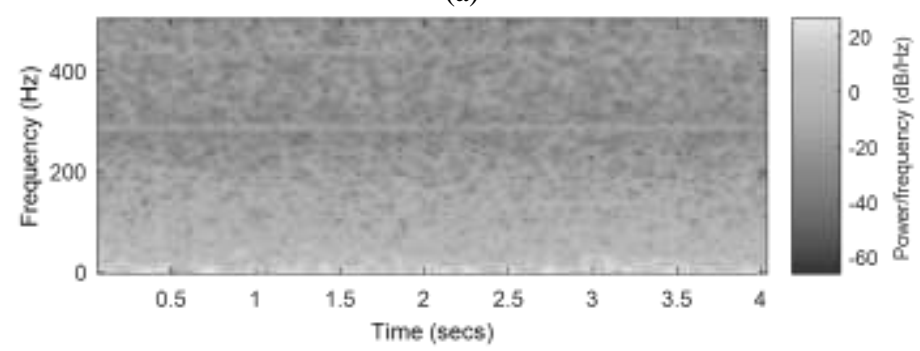

(b)

Fig. 2. a) EEG Signal with different amplitude along time, b) EEG signal Spectrogram. 


\subsubsection{Gray-Level Co-occurrence Matrix (GLCM)}

GLCM is a statistical method for examining texture, which considers the spatial relationships between pixels. Statistical measures can be extracted with this method, such as contrast, correlation, and energy, among others. Different authors use these features and others for classification; in an analysis done by Mustafa et al., [2] to classify mental stages through spectrograms, the authors extracted 80 statistical features for four orientations of the matrix, reducing the features vector by applying PCA, and used K nearest neighbors $(\mathrm{KNN})$ to classify the stages. In [18], a comparison was made of two classifiers - Support Vector Machine and Artificial Neural Networks - following the same methodology, but the features vector had other statistical features, improving the accuracy for KNN (using Euclidian distance) in comparison with the Artificial Neural Network (ANN). A BCI system [19] based on motor imagery acts in real-time using a single channel to classify the left- and right-hand motor imagery signals; these features are texture descriptors, employing a logistic regression classifier in offline mode.

A methodology for detecting two different datasets —epileptic and sleep stages-is in [20]. By extracting statistical features of GLCM, these features were encoded using the Fisher Vector (FV) to be applied in an extreme machine learning for two different tests -in sleep stages and in epileptic stages. Another method implemented in [21] for classifying epileptic seizure electroencephalogram (EEG) signals uses three texture descriptors to extract features: GLCM, the texture feature coding method (TFCM), and local binary patterns (LBP). GLCM and TFCM provided statistical features and LBP used the histogram; SVM was employed with LIBLINEAR in the classification with different accuracies, obtaining the highest accuracy with LBP and GLCM. Automatic person authentication [7] uses statistical features from spectrograms, but the energy obtained is used as a feature as well; a sum of the distance of the features is calculated to reduce dimension, and finally, ANN and SVM are applied.

In [22], co-occurrences of histograms of oriented gradients (CoHOG) and $\mathrm{Eig}(\mathrm{Hess})-\mathrm{CoHOG}$ features are extracted from the spectrogram; the features obtained are used in a nonnegative least squares classifier (NNLS), to classify alcoholism and control EEG signals.

\subsubsection{Frequency Bands}

An EEG Signal is commonly described in terms of brain activities; these are divided into frequency bands, which have a certain biological significance and different properties. Delta $(\delta)$ has the highest amplitude and the slowest wave; it is associated with deep sleep and waking states. Theta $(\theta)$ has an amplitude greater than $20 \mu \mathrm{V}$ and a range of 4-7 Hz, and is linked with idling, creative inspiration, unconscious material, drowsiness, and deep meditation. Alpha $(\alpha)$ has an amplitude of $30-50 \mu \mathrm{V}$ and a range of 8$13 \mathrm{~Hz}$, it is usually associated with relaxation, concentration, and sometimes with attention. $\mathrm{Mu}(\mu)$, is found in the alpha wave and is regularly related to suppression, indicating that the motor neurons are working. Beta $(\beta)$ is linked to alertness, thinking, and active concentration, and falls in the range between 12 and $30 \mathrm{~Hz}$. Lastly, Gamma 
$(\gamma)$ with a frequency greater than $30 \mathrm{~Hz}$, is seen during short term memory matching of recognized objects, sounds, or tactile sensations [8].

The previous section showed different techniques for extracting features from a spectrogram based on GLCM. Some authors have obtained the spectrograms from a specific band or from several bands; however, features are extracted directly from GLCM. In this section, methods for extracting features based on spectrograms of different frequency bands are shown. A method based on these spectrograms [15] for comparing obtained components during writing and imagined writing is implemented; to obtain the spectrogram, the STFT is applied to EEG signals, and the resulting spectrograms are modified to have components only in the frequency domain. All components are compared employing a correlation, concluding that the writing and imagined writing features are the same.

The classification of an epileptic seizure [23] employs the extraction of Frequency Cepstral Coefficients and stochastic relevance analyses on spectrograms of different rhythms. SVM is applied, obtaining competitive accuracies for different datasets. On the other hand, [24] extracts Malmquist-Takenaka coefficients from Spectrograms and statistical features, with the same objective. In this methodology, STFT is in discrete form, and the classification uses an Alternating Decision Tree (ADTree) classifier with three different datasets.

Another work [25] identifies a motor imagery database of left- and right-hand movements, applying multivariate empirical mode decomposition (MEMD) to generate multiple intrinsic mode functions (IMFs) to the EEG recordings. STFT is applied to the most significant mode. Features such as peak and entropy of the magnitude spectrum are used for the KNN classifier. Another work that implements KNN is [26], using KdTrees in this case; the features are the energy of the spectrograms in different frequency bands, obtaining a different accuracy and varying the number of features; the aim of this work is to detect drowsiness automatically.

\section{Discussion}

The reviewed literature in previous sections shows methods implemented for analyzing EEG signals based on spectrograms, using STFT as a representation in time-frequency, due to the competitive results compared with analyses based on time or frequency domain, as well as representations. This section shows relevant characteristics of the techniques in EEG processing. Table 2 shows the analysis of different methodologies, with attributes such as authors (reference), type of dataset to classify (objective), a brief description of the data (information), (\#) the number of classes to use, and finally, three columns (preprocessing, feature extraction, and classification) to identify the techniques used in the analysis phases.

\subsection{EEG Analysis}

In section two, the steps to follow in the EEG analysis were presented. The first step removes artifacts and noise; a few experiments $[19,23,25]$ apply low-pass and band- 
pass filters, which can be implemented easily, as the band frequencies are known. Most experiments do not apply this phase, although the datasets were acquired from the experiments. As general information, the following was identified: the number of classes (column \#) that predominate for the analysis are only two, employing a maximum of three classes. Regardless of the approaches for the data sets, the method used for the preprocessing stage is filtering, which is applied in few experiments.

Table 2. Methods about EEG signals analysis based on spectrograms.

\begin{tabular}{|c|c|c|c|c|c|c|}
\hline Reference & Objective & Information & $\#$ & $\begin{array}{l}\text { Prepro- } \\
\text { cessing }\end{array}$ & $\begin{array}{l}\text { Feature } \\
\text { extraction }\end{array}$ & Classification \\
\hline $\begin{array}{l}\text { Mustafa et } \\
\text { al. } 2010 .\end{array}$ & $\begin{array}{l}\text { Image Classifi- } \\
\text { cation }\end{array}$ & Proposed Dataset & 3 & - & $\begin{array}{l}\text { Statistical features } \\
\text { and PCA. }\end{array}$ & KNN (70.83\%). \\
\hline $\begin{array}{l}\text { Mustafa et } \\
\text { al. 2012. }\end{array}$ & $\begin{array}{l}\text { Brain balancing } \\
\text { classification }\end{array}$ & Proposed Dataset & 3 & - & $\begin{array}{l}\text { Statistical features } \\
\text { and PCA. }\end{array}$ & $\begin{array}{l}90 \% \text { KNN Euclid- } \\
\text { ian distance) and } \\
87.5 \% \text { ANN. }\end{array}$ \\
\hline $\begin{array}{l}\text { Duque, et } \\
\text { al. } 2014\end{array}$ & $\begin{array}{l}\text { Epileptic sei- } \\
\text { zures. }\end{array}$ & $\begin{array}{l}\text { 100 Signals, single } \\
\text { channel, } 5 \text { da- } \\
\text { tasets, } 173.61 \mathrm{~Hz} \text {, } \\
\text { Bonn University. }\end{array}$ & 2 & Low-pass & $\begin{array}{l}\text { Stochastic analysis } \\
\text { and cepstrals coef- } \\
\text { ficients. }\end{array}$ & $\begin{array}{l}95.78 \%-100 \%, \\
\text { SVM. }\end{array}$ \\
\hline $\begin{array}{l}\text { Kovács, et } \\
\text { al, } 2014 .\end{array}$ & $\begin{array}{l}\text { Epileptic sei- } \\
\text { zures. }\end{array}$ & $\begin{array}{l}100 \text { Signals, sin- } \\
\text { gle channel, } 5 \mathrm{da}- \\
\text { tasets, } 173.61 \mathrm{~Hz} \text {, } \\
\text { Bonn University. }\end{array}$ & 2 & - & $\begin{array}{l}\text { Malmquist- } \\
\text { Takenaka coeffi- } \\
\text { cients and statisti- } \\
\text { cal features. }\end{array}$ & $\begin{array}{l}96.7 \%, 98.36 \%, \\
\text { and } 99.7 \% . \text { alter- } \\
\text { nating decision } \\
\text { tree }\end{array}$ \\
\hline $\begin{array}{l}\text { Khairul, } \\
\text { et al, } 2015\end{array}$ & Imagery motor. & $\begin{array}{l}1 \text { person, } 2 \text { chan- } \\
\text { nel, } 128 \mathrm{~Hz} \text {. }\end{array}$ & 2 & Band-pass & Threshold. & KNN $(90 \%)$ \\
\hline $\begin{array}{l}\text { Kumar } \\
\text { and } \\
\text { Sharma, } \\
2015\end{array}$ & $\begin{array}{l}\text { Epileptic sei- } \\
\text { zures. }\end{array}$ & $\begin{array}{l}100 \text { Signals, sin- } \\
\text { gle channel, } 5 \mathrm{da}- \\
\text { tasets, } 173.61 \mathrm{~Hz} \text {, } \\
\text { Bonn University. }\end{array}$ & 2 & - & Energy and PCA. & $\begin{array}{l}\text { ANN }(995 \%, \\
99.33 \% \text { and } \\
92.36 \%)\end{array}$ \\
\hline $\begin{array}{l}\text { Nieves } \\
\text { and Ma- } \\
\text { nian, } \\
\text { 2016. }\end{array}$ & Authentication. & $\begin{array}{l}\text { Easycap, } 32 \text { chan- } \\
\text { nels. C3,CZ,C4. } \\
\text { Imagery move- } \\
\text { ments. }\end{array}$ & 2 & - & $\begin{array}{l}\text { Energy and statisti- } \\
\text { cal features. Sum of } \\
\text { distances. }\end{array}$ & $\begin{array}{l}\text { ANN(6-channels)- } \\
98 \% \text {. SVM(2-3, } \\
\text { channels)-90\%, } \\
96 \% \text {. }\end{array}$ \\
\hline $\begin{array}{l}\text { Alcin, et } \\
\text { al, 2016 }\end{array}$ & $\begin{array}{l}\text { Multiclass } \\
\text { (sleep stages } \\
\text { and Epileptic } \\
\text { seizures) }\end{array}$ & $\begin{array}{l}100 \text { Signals, sin- } \\
\text { gle channel, } 5 \mathrm{da}- \\
\text { tasets, } 173.61 \mathrm{~Hz} \text {, } \\
\text { Bonn University. } \\
\text { Proposed dataset, } \\
100 \mathrm{~Hz}, 30 \mathrm{~s}, 8 \text { sub- } \\
\text { jects. }\end{array}$ & 2 & - & GLCM y VF. & $\begin{array}{l}\text { Extreme machine } \\
\text { learning. } \\
95.17 \%, 95.38 \% \text { - } \\
\text { sleep stages. } \\
96.40 \% \text {-Epilepsy. }\end{array}$ \\
\hline $\begin{array}{l}\text { Camacho, } \\
\text { et al. 2016. }\end{array}$ & $\begin{array}{l}\text { Imagery motors } \\
\text { in a BCI. }\end{array}$ & $\begin{array}{l}1 \text { channel, } 400 \mathrm{~Hz} \\
20 \text { subjects. }\end{array}$ & 2 & Band-pass & $\begin{array}{l}\text { GLCM. Statistical } \\
\text { features. }\end{array}$ & $\begin{array}{l}\text { Linear regression, } \\
87.6 \% \text {. }\end{array}$ \\
\hline $\begin{array}{l}\text { Jalilifard, } \\
\text { et al., 2016 }\end{array}$ & Drowsiness & 10 subjects. & 2 & - & $\begin{array}{lr}\text { Energy } & \text { statistical } \\
\text { features. } & \text { Random } \\
\text { Forest. } & \\
\end{array}$ & $\begin{array}{l}88.57 \%-91 \% \\
\text { (KNN-Kd Trees) }\end{array}$ \\
\hline $\begin{array}{l}\text { Bajaj, et } \\
\text { al.,2016 }\end{array}$ & $\begin{array}{l}\text { Alcoholism and } \\
\text { mental control. }\end{array}$ & $\begin{array}{l}120 \text { files, } 64 \text { elec- } \\
\text { trodes, } 256 \mathrm{~Hz} \text {. }\end{array}$ & 2 & - & $\begin{array}{l}\text { Gray scale, Co- } \\
\text { HOG } \\
\text { Eig(Hess)-CoHOG } \\
\text { Statistics. } \\
\end{array}$ & $\begin{array}{l}91.67 \%, 91.67 \% \\
\text { and } 95.83 \% \\
\text { (NNLS) }\end{array}$ \\
\hline $\begin{array}{l}\text { Segür, et } \\
\text { al., } 2016\end{array}$ & $\begin{array}{l}\text { Epileptic } \quad \text { sei- } \\
\text { zures. }\end{array}$ & $\begin{array}{l}100 \text { Signals, sin- } \\
\text { gle channel, } 5 \mathrm{da}- \\
\text { tasets, } 173.61 \mathrm{~Hz} \text {, } \\
\text { Bonn University. } \\
\text { Proposed dataset, } \\
100 \mathrm{~Hz}, 30 \mathrm{~s}, 8 \text { sub- } \\
\text { jects. }\end{array}$ & 2 & - & $\begin{array}{l}\text { GLCM, statistical } \\
\text { features; LBP y } \\
\text { TFCM. }\end{array}$ & $\begin{array}{l}\text { 100\%, SVM and } \\
\text { LIBLINEAR. }\end{array}$ \\
\hline
\end{tabular}

For the feature extraction, statistical values of the time-frequency representation are utilized; it was seen that feature selection methods were applied in only three experiments; some authors implemented this stage through PCA [2,3,27] [3], sum of distances 
[7], and random forest [26]. Finally, the classification was generally performed by KNN, SVM, and ANN; SVM was the best classifier in regard to accuracy.

The methods described are focused on medical applications related to sleep, aspects of authentication, the detection of imaginary motor images, and others. Medical applications are aimed at the detection of brain abnormalities within the EEG signals, such as neurological disorders caused by epileptic seizures, and the effects of alcohol on the brain. In sleep analysis, experiments are performed for the detection of sleep states to identify problems of drowsiness. However, applications aimed at aspects of authentication, show results that can be competitive compared to other biometrics, demonstrating that the EEG signal has the sufficient properties to make it safe, unique, non-visible, and non-modifiable.

Table 3. Features extracted from spectrograms.

\begin{tabular}{|c|c|c|}
\hline Reference & $\begin{array}{l}\text { Feature } \\
\text { extraction method }\end{array}$ & Features Obtained \\
\hline $\begin{array}{l}\text { Mustafa et al. } \\
2010 .\end{array}$ & $\begin{array}{l}\text { Statistical features and } \\
\text { PCA. }\end{array}$ & $\begin{array}{l}\text { Proposed features by Haralick and Soh: Autocorrelation, Contrast, Corre- } \\
\text { lation, Cluster prominence, Cluster shade, Dissimilarity, Energy, Entropy, } \\
\text { Homogeneity, Maximum probability, Variance, Sum average, Sum vari- } \\
\text { ance, Sum entropy, Different variance, Different entropy, Information of } \\
\text { correlation 1, Information of correlation 2, Inverse difference normalized, } \\
\text { and Inverse difference moment normalized. }\end{array}$ \\
\hline $\begin{array}{l}\text { Mustafa et al. } \\
\text { 2012. }\end{array}$ & $\begin{array}{l}\text { Statistical features and } \\
\text { PCA. }\end{array}$ & $\begin{array}{l}\text { Proposed features by Haralick, Soh and Clausi: Autocorrelation, Contrast, } \\
\text { Correlation, Cluster prominence, Cluster shade, Dissimilarity, Energy, } \\
\text { Entropy, Homogeneity, Maximum probability, Variance, Sum average, } \\
\text { Sum variance, Sum entropy, Different variance, Entropy difference, In- } \\
\text { formation of correlation 1, Information of correlation 2, Inverse difference } \\
\text { normalized, and Inverse difference moment normalized. }\end{array}$ \\
\hline $\begin{array}{l}\text { Duque, et al. } \\
2014\end{array}$ & $\begin{array}{l}\text { Stochastic analysis and } \\
\text { cepstral coefficients. }\end{array}$ & $\begin{array}{l}\text { Five Cepstral coefficients in frequency related to rhythms: alpha, beta, } \\
\text { theta and delta. }\end{array}$ \\
\hline $\begin{array}{l}\text { Kovács, et al, } \\
\text { 2014. }\end{array}$ & $\begin{array}{l}\text { Malmquist-Takenaka } \\
\text { coefficients and statisti- } \\
\text { cal features. }\end{array}$ & $\begin{array}{l}\text { Features using the discrete STFT as from rational coefficients and five } \\
\text { statistical values of the coefficients: Mean median, maximum, minimum, } \\
\text { and standard deviation. }\end{array}$ \\
\hline $\begin{array}{l}\text { Khairul, et al, } \\
2015\end{array}$ & Threshold. & Peak and entropy of the magnitude spectrum. \\
\hline $\begin{array}{l}\text { Kumar and } \\
\text { Sharma, } 2015\end{array}$ & Energy and PCA. & Fractional energy for EEG segments. \\
\hline $\begin{array}{l}\text { Nieves y Ma- } \\
\text { nian, 2016. }\end{array}$ & $\begin{array}{l}\text { Energy and statistical } \\
\text { features. Sum of dis- } \\
\text { tances. }\end{array}$ & Energy, mean, variance and skewness from spectrogram. \\
\hline $\begin{array}{l}\text { Alcin, et al, } \\
2016\end{array}$ & GLCM y VF. & Texture descriptors: Contrast, correlation, energy, and homogeneity. \\
\hline $\begin{array}{l}\text { Camacho, et } \\
\text { al. 2016. }\end{array}$ & $\begin{array}{l}\text { GLCM. Statistical fea- } \\
\text { tures. }\end{array}$ & $\begin{array}{l}\text { Texture descriptor: Correlation, energy, contrast, homogeneity, and dis- } \\
\text { similarity. }\end{array}$ \\
\hline $\begin{array}{l}\text { Jalilifard, et } \\
\text { al., } 2016\end{array}$ & $\begin{array}{l}\text { Energy statistical fea- } \\
\text { tures. Random Forest }\end{array}$ & $\begin{array}{l}\text { The mean value of power in time and standard deviation (SD) and Shanon } \\
\text { entropy related to each time-segment were computed from the time-do- } \\
\text { main. }\end{array}$ \\
\hline $\begin{array}{ll}\text { Bajaj, } \\
\text { al.,2016 }\end{array}$ & $\begin{array}{l}\text { Gray scale, CoHOG } \\
\text { and Eig(Hess)-CoHOG } \\
\text { Statistics. }\end{array}$ & $\begin{array}{l}\text { Features obtained from local texture: Co-occurrence of Oriented Gradient } \\
(\mathrm{CoHOG}) \text { and Eig(Hess). }\end{array}$ \\
\hline $\begin{array}{l}\text { Segür, et al., } \\
2016\end{array}$ & $\begin{array}{l}\text { GLCM, Statistical fea- } \\
\text { tures, LPB and TFCM. }\end{array}$ & Texture descriptors: Contrast, correlation, energy, and homogeneity. \\
\hline
\end{tabular}

From the different, it was noted that for the analysis of epilepsy diseases, there is a set of data that different authors [3,20,23,24] employ, for example use a dataset created by the University of Bonn with five different subsets (A-E), with 100 single channel 
signals, a duration of 26.3 seconds and a sampling frequency of $173.61 \mathrm{~Hz}$. This database contains high quality information (number of studies, time and number of seizures), which were validated by experts. Table 2 shows four works where this dataset was used; although only one implemented the preprocessing stage [23] and the accuracy obtained was between $95.78 \%$ and $100 \%$.

\subsection{STFT and Features}

Different objectives are proposed in the analyzed works, such as classification of motor images, the detection of imagined words, and others; nevertheless, they do not show execution time, which could be due to the real-time application execution not being required. STFT implementation is fast, but resolution depends on the window selected. For the purposes of the experiments in the literature, the resolution obtained with STFT is enough, implying less computational work and less execution time. However, it was shown in $[2,4,5]$ that dimensionality is a problem to be solved; experiments where PCA was used do not report execution time and [22] do not apply reduction of characteristics, although they do note that it is necessary to reduce the vectors, as they have thousands of values.

All works use different features, most of them based on texture; some works extract features from GLCM applied to spectrograms; although the features are similar, changing some of these affect accuracy. Table 3 shows features extracted from spectrograms by the reviewed analysis; the first column corresponds to the authors, the second shows the methods implemented for extraction, and the last presents the features obtained.

These works generally extract features from texture or from statistics based on spectrograms, while others use energy as their main feature and employ different techniques, such as LBP, TFCM, CoHOG, and Cepstral coefficients, among others. Different methods are implemented; however, the most widely used is based on texture descriptors and energy.

\section{Conclusions}

The Short-Time Fourier Transform provides a quick implementation for different approaches; acceptable results have been reported in terms of classification, derived from an extraction of spectrogram features obtained by applying STFT to the EEG signal. However, despite the results in the literature with this representation, there is a limited scope, considering the complexity of the characteristics, which presents different research challenges, such as computational performance, accuracy in the classification, data dimension reduction, filtering of noisy signals, and others. Therefore, it is necessary to propose improved methodologies that can meet these challenges in all phases of analysis.

In some experiments, spectrograms have shown low resolution, which implies a changing performance; however, a high resolution requires more computational work and time. To solve this, a different purpose of feature extraction can be proposed, in addition to a feature selection method, because of the large dimensional data vector. 
Due the information provided by spectrograms, it is possible to apply a classification based on thresholds. The literature on this methodology does not usually explain how the threshold is obtained, but another way to solve this, could be through the implementation of genetic algorithms.

Most of the methodologies here presented analyze a single dataset as a seizure, a dream, alcoholism, imaginary movements, or others, but only one of these at a time; however, a different methodology could be proposed to classify more than one EEG signal. [20] classifies two datasets with competitive results in terms of accuracy, compared with methodologies whose results come from one analysis to a single dataset.

\section{References}

1. Ridouh, A., Boutana, D., Benidir, M.: Comparative Study of Time Frequency Analysis Application on Abnormal EEG Signals. Recent Advances in Electrical Engineering and Control Applications, Springer International Publishing, Cham 355-368 (2017)

2. Mustafa, M., Taib, M., Murat, Z., Sulaiman, N., Aris, S.: The Analysis of EEG Spectrogram Image for Brainwave Balancing Application Using ANN. In: 2011 UkSim 13th International Conference on Computer Modelling and Simulation, 64-68 (2011)

3. Kumar, J., Bhuvaneswari, P.: Analysis of Electroencephalography (EEG) Signals and Its Categorization-A Study. Procedia Engineering, 38, 2525-2536 (2012)

4. Ilyas, M., Saad, P., Ahmad, M.: A survey of analysis and classification of EEG signals for brain-computer interfaces. In: 2015 2nd International Conference on Biomedical Engineering (ICoBE), 1-6 (2015)

5. Gandhi, V.: Chapter 2 - Interfacing Brain and Machine. Brain-Computer Interfacing for Assistive Robotics, Academic Press, San 7-63 (2015)

6. Grossi, E., Olivieri, C., Buscema, M.: Diagnosis of autism through EEG $\backslash$ processed by advanced computational algorithms: A pilot study. Computer Methods and Programs in Biomedicine, 142, 73-79 (2017)

7. Nieves, O., Manian, V.: Automatic person authentication using fewer channel EEG motor imagery. In: 2016 World Automation Congress (WAC), 1-6 (2016)

8. Ramadan, R., Vasilakos, A.: Brain computer interface: control signals review. Neurocomputing, 223, 26-44 (2017)

9. Alamdari, N., Haider, A., Arefin, R., Verma, A., Tavakolian, K., Fazel-Rezai, R.: A review of methods and applications of brain computer interface systems. In: 2016 IEEE International Conference on Electro Information Technology (EIT), 345-350 (2016)

10. Al-Ani, A., Koprinska, I., Naik, G., Khushaba, R.: A dynamic channel selection algorithm for the classification of EEG and EMG data. In: 2016 International Joint Conference on Neural Networks (IJCNN), 4076-4081 (2016)

11. Ren, W., Han, M., Wang, J., Wang, D., Li, T.: Efficient feature extraction framework for EEG signals classification. In: 2016 Seventh International Conference on Intelligent Control and Information Processing (ICICIP), 167-172 (2016)

12. Li, Y., Wang, X., Luo, L., Li, K., Yang, X., Guo, Q.: Epileptic Seizure Classification of EEGs using Time-Frequency Analysis based Multiscale Radial Basis Functions. IEEE Journal of Biomedical and Health Informatics 1-12 (2017)

13. Wang, Y., Xu, G., Zhang, S., Luo, A., Li, M., Han, C.: EEG \signal co-channel interference suppression based on image dimensionality reduction and permutation entropy. Signal Processing, 134, 113-122 (2017) 
14. Lakshmi, M., Prakash, C.: Survey on EEG Signal Processing Methods (2014)

15. Zabidi, A., Mansor, W., Lee, Y., Fadzal, C.: Short-time Fourier Transform analysis of EEG signal generated during imagined writing. In: 2012 International Conference on System Engineering and Technology (ICSET), 1-4 (2012)

16. Boashash, B., ed.: Chapter 2 - Heuristic Formulation of Time-Frequency Distributions. Time-Frequency Signal Analysis and Processing, Second Edition, Academic Press, Oxford, 65-102 (2016)

17. El-Shennawy, K.: Communication Theory and Signal Processing for Transform Coding. Bentham Science Publishers (2014)

18. Mustafa, M., Taib, M., Murat, Z., Sulaiman, N.: Comparison between KNN and ANN Classification in Brain Balancing Application via Spectrogram Image. Journal of Computer Science \& Computational Mathematics, 2(4), 17-22 (2012)

19. Camacho, J., Manian, V.: Real-time single channel EEG motor imagery based Brain Computer Interface. In: 2016 World Automation Congress (WAC), 1-6 (2016)

20. Alçin, Ö., Siuly, S., Bajaj, V., Guo, Y., Şengür, A., Zhang, Y.: Multi-category EEG $\backslash$ signal classification developing time-frequency texture features based Fisher Vector encoding method. Neurocomputing, 218, 251-258 (2016)

21. Şengür, A., Guo, Y., Akbulut, Y.: Time--frequency texture descriptors of EEG signals for efficient detection of epileptic seizure. Brain Informatics, 3, 101-108 (2016)

22. Bajaj, V., Guo, Y., Sengur, A., Siuly, S., Alcin, O.: A hybrid method based on timefrequency images for classification of alcohol and control EEG signals. Neural Computing and Applications, 1-7 (2016)

23. Duque-Muñoz, L., Espinosa-Oviedo, J., Castellanos-Dominguez, C.: Identification and monitoring of brain activity based on stochastic relevance analysis of short-time EEG rhythms. BioMedical Engineering OnLine, 13, 123 (2014)

24. Kovacs, P., Samiee, K., Gabbouj, M.: On application of rational Discrete Short Time Fourier Transform in epileptic seizure classification. In: 2014 IEEE International Conference on Acoustics, Speech and Signal Processing (ICASSP), 5839-5843 (2014)

25. Bashar, S., Hassan, A., Bhuiyan, M.: Motor imagery movements classification using multivariate EMD and short time Fourier transform. In: 2015 Annual IEEE India Conference (INDICON), 1-6 (2015)

26. Jalilifard, A., Pizzolato, E.: An efficient K-NN approach for automatic drowsiness detection using single-channel EEG recording. In: 2016 38th Annual International Conference of the IEEE Engineering in Medicine and Biology Society (EMBC), 820-824 (2016)

27. Deng, L., OŚhaughnessy, D.: Speech Processing: A Dynamic and Optimization-Oriented Approach. Taylor \& Francis (2003) 